\title{
On (finite) distributive lattices with antitone involutions
}

\author{
Jan Kühr \\ (joint work with Michal Botur) \\ Department of Algebra and Geometry, \\ Faculty of Science, Palacký University in Olomouc, \\ 17. listopadu 12, CZ-77146 Olomouc, Czech Republic \\ jan.kuhr@upol.cz
}

Some well-known algebras of logic or other algebraic models of non-classical reasoning, such as MV-algebras or orthomodular lattices, can be regarded as lattices with antitone involutions, i.e., bounded lattices with the property that all principal filters (or ideals) are equipped with antitone involutions. This simple observation is behind the so-called "basic algebras" that were introduced in 1 in an attempt to generalize orthomodular lattices in a similar way in which $\mathrm{MV}$-algebras generalize boolean algebras. Roughly speaking, a basic algebra is an algebra $\langle A ; \oplus, \neg, 0,1\rangle$ of type $\langle 2,1,0,0\rangle$ such that the rule $x \leq y$ iff $\neg x \oplus y=1$ defines a bounded lattice in which, for every $a \in A$, the map $f_{a}: x \mapsto \neg x \oplus a$ is an antitone involution on the interval $[a, 1]$, and where $\neg x=f_{0}(x)$ and $x \oplus y=f_{y}(\neg x \vee y)$ for all $x, y \in A$. MV-algebras then coincide with the associative (and commutative) basic algebras, and orthomodular lattices with those satisfying the quasi-identity $x \leq y \Rightarrow y \oplus x=y$.

In general, we cannot say much about the underlying lattice of a basic algebra, but in several important particular cases, when the antitone involutions satisfy certain natural conditions, the lattice is distributive. Therefore, we focus on distributive basic algebras, with emphasis on the structure of finite ones.

The main objective for the first part is to prove that the underlying lattice of a finite distributive basic algebra is a direct product of chains, though the algebra itself need not be an $\mathrm{MV}$-algebra. This means that all finite distributive basic algebras can be obtained from finite MV-algebras by "perturbing" the standard pointwise "MV-involutions". In the second part we consider algebras satisfying the identity $x \leq x \oplus y$, which is the most general version of the aforementioned natural conditions, because it actually says that $f_{0}(x) \leq f_{a}(x)$ for all $a \leq x$. Among others, we prove that these basic algebras are distributive and in the finite case they are just MV-algebras. Finally, in the third part we deal with lattice effect algebras that play an important role in investigating the logical foundations of quantum mechanics and can also be identified with certain basic algebras. Besides proving that every lattice effect algebra satisfying $x \leq x \oplus y$ is an MV-algebra, we focus on the variety of distributive lattice effect algebras.

\section{References}

[1] I. Chajda, R. Halaš, and J. Kühr: Many valued quantum algebras, Algebra Univers. 60 (2009) 63-90.

[2] I. Chajda and J. Kühr: Finitely generated varieties of distributive effect algebras, Algebra Univers. 69 (2013) 213-229. 